\title{
Analysis of Association Between MGMT and p53 Gene Single Nucleotide Polymorphisms and Laryngeal Cancer
}

\author{
YAYUN LV ${ }^{1,2 *}$, CHUANLIANG JIA ${ }^{2 *}$, AIHUA JIANG ${ }^{3}$, HUA ZHANG $^{2}$, YUNQIANG WANG ${ }^{4}$, \\ FEIFEI LIU ${ }^{2}$, LINLIN YANG ${ }^{2}$, YAN SUN ${ }^{2}$, RUNLI LV ${ }^{5}$ and XICHENG SONG ${ }^{2}$ \\ ${ }^{1}$ Binzhou Medical University, Yantai, P.R. China; \\ Departments of ${ }^{2}$ Otorhinolaryngology and Head and Neck Surgery, ${ }^{3}$ Anesthesia, ${ }^{4}$ Imaging, \\ and ${ }^{5}$ Surgery, Yuhuangding Hospital of Qingdao University, Yantai, P.R. China
}

\begin{abstract}
Aim: To investigate the $p 53$ and $O^{6}$ methylguanine DNA methyltransferase (MGMT)5' upstream sequence gene promoter regions for single nucleotide polymorphisms and explore the p53 gene 5' upstream sequence consisting of two haplotypes to provide a genetic marker for the incidence of laryngeal squamous cell carcinoma. Materials and Methods: We included 96 cases of laryngeal squamous cell carcinoma and 102 controls. We used SNaPshot micro-sequencing analysis of the MGMT promoter region for four single nucleotide polymorphisms and p53 gene 5' upstream sequence loci (rs1625649, rs2287499, rs2287498, rs228749) genotypes. We calculated and compared two groups for genotypic and allelic frequencies, applied HaploView4.2 for computing rs2287499, rs2287498, rs228749 values and haplotype frequencies and tested control loci and Hardy-Weinberg equilibrium. All the experimental data were statistically evaluated using SPSS17.0. The Chi-square test was used for statistical analysis with $p<0.05$ indicating statistical significance. Results: 5'Upstream single nucleotide polymorphisms rs1625649, rs2287499, rs2287498, rs228749 of p53 were polymorphic in both patient and control groups. There was no statistical significance in frequency distributions for the four loci genotypes when comparing patients and healthy controls (Chi-square values were 4.47, 0.98, 1.67, 4.68, respectively; $p>0.05$ ). However,
\end{abstract}

*These Authors contributed equally to this study.

Correspondence to: Song Xicheng, MD, Ph.D., Department of Otorhinolaryngology and Head and Neck Surgery, Yuhuangding Hospital of Qingdao University No 20 East Yuhuangding Road, Yantai 264000, PR China. E-mail: songxicheng@126.com

Key Words: $M G M T, p 53$, single nucleotide polymorphism, laryngeal cancer, laryngeal squamous cell carcinoma. allelic frequencies of the MGMT promoter region locus rs 1625649 between patients and healthy control groups were statistically significantly different (chi-square value of 5.77; $p$ <.05). Differences between allelic frequencies for the p53 gene 5' upstream sequence loci rs2287499, rs2287498 and rs228749 between patients and the healthy control group were not statistically significant (Chi-square values were $1.11,1.56,3.36 ; p>0.05)$. Nor were those for the two haplotypes of $r s 2287499, r s 2287498$ and $r s 228749$ between patients and the healthy control group were not statistically significant (Chi-square value 1.46, p>0.05). Conclusion: MGMT gene polymorphism appears to be associated with the incidence of laryngeal cancer.

Most laryngeal cancers are squamous cell carcinomas, reflecting their origin from the squamous cells which form the majority of the laryngeal epithelium. Smoking is the most important risk factor for laryngeal cancer. Death from laryngeal cancer is 20 times more likely for heavy smokers than for nonsmokers (1). Despite many individuals being exposed to environmental or lifestyle risk factors, laryngeal cancer does not develop in all people exposed, suggesting a genetic susceptibility for developing this malignancy. Tobacco carcinogens such as alkylating agents can lead to mismatch including $O^{6}$-methylguanineDNA $\left(O^{6}-\mathrm{MeG}\right)$ which is highly harmful. $O^{6}$-Methylguanine DNA methyltransferase (MGMT) plays an important role in the repair of $O^{6}-\mathrm{MeG}(2-4)$. The tumor suppressor $p 53$ is a critical factor in cell-cycle control and apoptosis, and its loss of function has been shown to play a pivotal role in cancer development and progression. The G/T p53 mutation is the most common type of base mutation, with active expression of $M G M T$ able to prevent the mutation of $\mathrm{G}: \mathrm{C}$ to $\mathrm{A}: \mathrm{T}$.

SNapshot microsequencing analysis was adopted in this study to investigate $p 53$ and $M G M T$ polymorphism. Preliminary statistical analysis was carried out on samples 
from patients with laryngeal cancer and healthy controls in the Yantai area of Shandong province in order to investigate the correlation of the four loci with laryngeal carcinoma in a Yantai Han population, and to provide a theoretical basis for the prevention and treatment of laryngeal carcinoma $(5,6)$.

\section{Materials and Methods}

Patients. From November 2012 to December 2013, 96 male patients with laryngeal cancer at Yantai Yuhuangding Hospital were selected for the study. The average age of patients was $53 \pm 13$ years and their clinical status was confirmed by pathological diagnosis. The control group consisted of unrelated male patients without tumor selected at the same time, with an average age of $55 \pm 9$ years. Patients included in the study gave their informed consent before commencement of the study: the controls had no history of cancer, no history of laryngeal precancerous lesions (including leukoplakia of the larynx, laryngeal pachydermia, laryngeal papilloma,chronic hypertrophic laryngitis), no congenital genetic history and no history of blood transfusion within the previous 3 months.

We selected four polymorphic sites from The Single Nucleotide Polymorphism Database (dbSNP) and Haplotypemap (HapMap Genome Browser release\#24; phase 1 and 2-full dataset, ftp://ftp.ncbi.nlm.nih.gov/hapmap/) for human MGMT and p53 genes at the 5 'end. Screening conditions were that the site had to be located on the 5' gene-regulatory region; individuals were from the Han population in Beijing China (Han Chinese in Beijing, CHB) with a minor allelic frequency $\geq 10 \%$; the polymorphisms must have been associated with tumor in published studies. We searched for these four polymorphisms via the Human Genome Variation Society Mutation Research Society, the human genome, to ensure that the relevant research literature could be obtained.

Peripheral blood $(3 \mathrm{ml})$ was obtained from each participant, using EDTA anticoagulation collection. Genomic DNA was extracted from white blood cells with phenol/chloroform extraction. The primers used in this study (according to Primer Extension Kit SNaPshot operation, completed by the United States ABI 3130 sequencing(Thermo Fisher Scientific, Shanghai, China) were as follows: MGMT rs1625649 upstream amplification primers 5'TGCCCGAGTGGTCCTGAAAG-3', downstream amplification primers 5'-CTCTGCTGGTCTGGGGGTCC-3', extension primers 5'-tttttt tttttGAGTCTCTCCCCTCCTGG-3'. rs2287497 upstream amplification primers 5'-TGCTTTTCA AGTGTAGGGCTAG-3', downstream amplification primers 5'-GCACGTAGCCCTTTTAGA CTGA-3', extension primers 5'-tttttttttttttttttttttttAGACTGAG CTTACATTTT(A/G)TCTA-3; rs2287498 upstream amplification primers 5'-TGAAAATCTCGG GGGTGGTC-3, downstream amplification primers 5'-GGGAGATGAAGTGTGAGGTCG-3, extension primers $5^{\prime}$ - $-\mathrm{ttttt} t \mathrm{t}$ ttttttttttt $\mathrm{ttt}$ AAA TCGAGGC AGCTGGGA-3'; rs2287499 upstream amplification primers 5'GGCTTTTC CAGAC C CCAACT-3', downstream amplification primers 5'-CGGACTCTGAACTGATGCCAC-3', amplification primers 5'-ttttttttttttttCAGCT(G/A)TGTCCCAGG AGCTA-3'. GeneMapper 4.0 (http://genemapper.software.informer.com/4.0/) automatically read the results obtained from the sequencing instrument, with manual inspection correction. HaploView4.2 (http://www.broadinstitute.org) analysis was used to study the degree of linkage and haplotype. In order to test the samples met
Table I. Data on polymorphisms.

\begin{tabular}{lcccc}
\hline dbSBP & $\begin{array}{c}\text { Chromosomal } \\
\text { location }\end{array}$ & MAF & $\begin{array}{c}\text { Variant } \\
\text { base }\end{array}$ & Gene \\
\hline rs1625649 & 131264931 & 0.3852 & $\mathrm{G} / \mathrm{T}$ & MGMT \\
rs2287499 & 7592168 & 0.3379 & $\mathrm{C} / \mathrm{G}$ & TP53 \\
rs2287498 & 7592560 & 0.1837 & $\mathrm{~A} / \mathrm{G}$ & TP53 \\
rs2287497 & 7592780 & 0.2801 & $\mathrm{C} / \mathrm{T}$ & TP53 \\
\hline
\end{tabular}

dbSBP: https://www.ncbi.nlm.nih.gov/projects/SNP/; MAF: minor allelic frequency.

the general population distribution, the Hardy-Weinberg equilibrium test was carried out on each single nucleotide polymorphisms (SNP). In both cases and controls, the frequencies of genotype and alleles were compared using the chi-square test, and statistical significance was defined as $p<0.05$. All the statistical analysis was carried out using SPSS17.0 (SPSS China, Shanghai, China).

\section{Results}

We screened the $M G M T$ promoter region loci rs1625649 and the p53 5' upstream sequences loci rs2287499, rs2287498 and rs2287497. The polymorphisms seen were rs1625649TT, rs1625649GT, rs1625649GG, rs2287499CC, rs2287499CG, rs2287499GG, rs2287498AA, rs2287498GA, rs2287498GG, rs2287497GG, rs2287497GA and rs2287497AA (Table I). The detection rate was $100 \%$. The allelic frequencies of the control group were consistent with Hardy-Weinberg equilibrium (Table II) and regarded as the natural state of the population. The distribution of rs1625649, rs2287499, rs2287498 and rs2287497 genotypes in the case and control groups were not statistically significant (Chi-square: 4.47, $0.98,1.67$ and $4.68 ; p>0.05$; Table III). However, the frequency of the rs1625649 allele in the case and control groups was statistically different (Chi-square 5.77; $p<0.05$; Table IV). The rs2287499, rs2287498 and rs2287497formed a haplotype block (Figure 1), composed of two haplotypes; the haplotypic frequencies were 0.678 and 0.322 . The distribution of the two haplotypes in the case and the control groups were not statistically significant (chi-square 1.46; $p>0.05$; Table V).

\section{Discussion}

Laryngeal cancer has been shown to be related to smoking, drinking, long-term inhalation of harmful substances, and infection among others $(7,8)$, which is exacerbated by the combustion of tobacco, slow ciliary movement, mucosal edema and hemorrhage. The thickened epithelial hyperplasia and squamous metaplasia provide an ideal foundation for 
Table II. Hardy-Weinberg equilibrium test.

\begin{tabular}{lccccc}
\hline Polymorphism & Recessive homozygote, $\mathrm{n}$ & Heterozygote, $\mathrm{n}$ & Dominant homozygote, $\mathrm{n}$ & Total & $p$-Value \\
\hline rs1625649 & 8 & 41 & 53 & 102 & 0.99 \\
rs2287499 & 11 & 36 & 55 & 102 & 0.41 \\
rs2287498 & 13 & 41 & 48 & 102 & 0.67 \\
rs2287497 & 7 & 34 & 61 & 102 & 0.76 \\
\hline
\end{tabular}

$\mathrm{n}$, Number of individuals.

Table III. Genotypic frequency statistical results.

\begin{tabular}{|c|c|c|c|c|}
\hline Polymorphism & $\begin{array}{c}\text { Cases, } \\
\mathrm{n}\end{array}$ & $\begin{array}{c}\text { Controls, } \\
\mathrm{n}\end{array}$ & Chi-square & $p$-Value \\
\hline \multicolumn{5}{|l|}{ rs1625649 } \\
\hline $\mathrm{TT}$ & 15 & 8 & 4.47 & 0.1 \\
\hline GT & 43 & 41 & & \\
\hline GG & 38 & 53 & & \\
\hline \multicolumn{5}{|l|}{ rs2287499 } \\
\hline GG & 14 & 11 & 0.98 & 0.61 \\
\hline $\mathrm{GC}$ & 36 & 36 & & \\
\hline $\mathrm{CC}$ & 46 & 55 & & \\
\hline \multicolumn{5}{|l|}{ rs2287498 } \\
\hline AA & 10 & 13 & 1.67 & 0.43 \\
\hline GA & 32 & 41 & & \\
\hline GG & 54 & 48 & & \\
\hline \multicolumn{5}{|l|}{ rs2287497 } \\
\hline AA & 16 & 7 & 4.68 & 0.09 \\
\hline GA & 30 & 34 & & \\
\hline GG & 50 & 61 & & \\
\hline
\end{tabular}

n, Number of individuals

cancer progression. Our study involved exclusively male patients as there were fewer female drinkers and smokers available for recruitment.

Tobacco-generated alkylating carcinogens cause alkylation damage to DNA bases of which $O^{6}-\mathrm{MeG}$ represents the greatest threat by causing G/T mismatch, which induces cell apoptosis or mutation (9). MGMT transfers the $O^{6}$ alkylating group from $O^{6}-\mathrm{MeG}$ to cysteine residues of its own molecule, repairing the guanine DNA chain damage. At the same time, MGMT irreversibly loses activity; therefore, the level of intracellular MGMT is positively correlated with the level of DNA damage that can be tolerated. In this study, we selected a $M G M T$ polymorphism closely related to laryngeal cancer p53 gene mutation as our research object, combined with p53 gene polymorphism analysis.
Table IV. Allelic frequency statistical results.

\begin{tabular}{lcccc}
\hline & $\begin{array}{c}\text { Cases, } \\
\mathrm{n}\end{array}$ & $\begin{array}{c}\text { Controls, } \\
\mathrm{n}\end{array}$ & Chi-square & $p$-Value \\
\hline $\begin{array}{l}\text { rs1625649 } \\
\text { T }\end{array}$ & 73 & 57 & & \\
$\quad \mathrm{G}$ & 119 & 147 & 5.77 & 0.01 \\
$\mathrm{rs} 2287499$ & & & & \\
$\mathrm{G}$ & 64 & 58 & & \\
$\mathrm{C}$ & 128 & 146 & 1.11 & 0.29 \\
$\mathrm{rs} 2287498$ & & & & \\
$\mathrm{~A}$ & 52 & 67 & 1.56 & 0.21 \\
$\mathrm{G}$ & 140 & 137 & & \\
rs2287497 & & & & \\
$\mathrm{A}$ & 61 & 48 & 3.36 & 0.06 \\
$\mathrm{G}$ & 131 & 156 & & \\
\hline
\end{tabular}

n, Number of alleles.



Figure 1. rs2287499, rs2287498, rs2287497 form a haplotype block, the two haplotypes are GGC and CAT.

Many phenotypic differences in human susceptibility to drugs or diseases may be related to SNPs (10) which are not genetically independent but tend to be heritable. Genetic SNP loci rarely undergo recombination during the genetic process, a group of SNPs called haplotype (11). We 
Table V. Haplotype frequency statistics.

\begin{tabular}{|c|c|c|}
\hline & Haplotype 1 (GGC), n & Haplotype 2 (CAT), n \\
\hline Cases & 63 & 33 \\
\hline Controls & 59 & 44 \\
\hline Total & 121 & 77 \\
\hline Chi-square & \multicolumn{2}{|c|}{1.46} \\
\hline$p$-Value & \multicolumn{2}{|c|}{0.22} \\
\hline
\end{tabular}

$\mathrm{n}$, Number of individuals.

studied the three $p 53$ gene loci rs2287499, rs2287498 and rs228797 which form a haplotype block, comprising two haplotypes. Statistical analysis showed that neither the SNP genotype nor haplotype was correlated consistently with laryngeal cancer. Although the relationship between these four polymorphisms and tumor has been studied (12-15), the polymorphisms in different ethnic groups are differently distributed. Studies outside China cannot be directly applied to the Chinese people. For example, the $M G M T$ polymorphism rs 16906252 containing $\mathrm{T}$ base and $M G M T$ promoter methylation as studied by Ogino et al., (16), which results in the prediction of SNP gene promoter methylation as a feasibility study cannot be applied. However, in the Millennium Genome Project browser, there appear to be no instances of rs 16906252 containing the $\mathrm{T}$ genotype. Genetic balance cannot be achieved under the natural state, but in a sufficiently large population, if the individual is free to reproduce and no obvious natural selection occurs, this tends to be seen as consistent with genetic equilibrium. In the control population included in this study, the Hardy-Weinberg equilibrium test values of four sites were greater than 0.05 , indicating that the population can be approximated as being in a natural state.

This study showed that the MGMT promoter region of rs 1625649 and $p 53$ gene 5' upstream sequences rs2287499, rs2287498 and rs2287497 genotypes were not statistically significantly distributed in the case and control groups, but the frequency of the MGMT rs1625649 allele was statistically significantly different, as were the haplotypes. These results may be due to population differences and the sample size not being large enough, but we should not exclude the possibility of other polymorphisms of $M G M T$ and $p 53$. As the sample is small, and given the relationship between SNPs and the incidence of laryngeal cancer to smoking and drinking status, we did not use multiple regression analysis. Therefore, it is necessary to further expand the sample size and further study other polymorphisms of $M G M T$ and p53.

\section{Acknowledgements}

This study was financially supported by Province Natural Foundation of Shandong (ZR2014HL079); Yantai municipal scientific and technological development project, China (No: 2014WS001); Yantai municipal scientific and technological development project, China(No: 2016WS010); Yantai municipal scientific and technological development project, China (No: 2016WS016); Yantai Yuhuangding Hospital Youth Scientific Research Fund, China (No:201610).

\section{References}

1 Ramroth H, Dietz A and Becher H: Environmental tobacco smoke and laryngeal cancer: results from a population-based case-control study. Eur Arch Oto-Rhino-Laryngol 265(11): 1367-1371, 2008.

2 Barton MB, Keane TJ and Gadalla T: The effect of treatment time and treatment interruption on tumour control following radical radiotherapy of laryngeal cancer. Radiother Oncol 23(3): 137-143, 1992.

3 Hillman RE, Walsh MJ and Wolf GT: Functional outcomes following treatment for advanced laryngeal cancer. Part I--Voice preservation in advanced laryngeal cancer. Part II-Laryngectomy rehabilitation: the state of the art in the VA System. Research Speech-Language Pathologists. Department of Veterans Affairs Laryngeal Cancer Study Group. Ann Otol Rhinol Laryngol (Suppl) 172: 1-27, 1998.

4 Paluszczak J, Misiak $\mathrm{P}$ and Wierzbicka M: Frequent hypermethylation of DAPK, RARbeta, MGMT, RASSF1A and FHIT in laryngeal squamous cell carcinomas and adjacent normal mucosa. Oral Oncol 47(2): 104-107, 2011.

5 Stransky N, Egloff AM and Tward AD: The mutational landscape of head and neck squamous cell carcinoma. Science 333(6046): 1157-1160, 2011.

6 Xu M, Nekhayeva I, Cross CE, Rondelli CM, Wickliffe JK and Abdel-Rahman SZ: Influence of promoter/enhancer region haplotypes on $M G M T$ transcriptional regulation: a potential biomarker for human sensitivity to alkylating agents. Carcinogenesis 35(3): 564-571, 2014.

7 Elwood JM, Pearson JCG, Skippen DH and Jackson SM: Alcohol, smoking, social and occupational factors in the aetiology of cancer of the oral cavity, pharynx and larynx. Int $\mathrm{J}$ Cancer 34(5): 603-612, 1984

8 Muscat JE and Wynder EL: Tobacco, alcohol, asbestos, and occupational risk factors for laryngeal cancer. Cancer 69(9): 2244-2251, 1992.

9 Meng HX, Hackett JA and Nestor C: Apoptosis and DNA methylation. Cancers 3(2): 1798-1820, 2011.

10 Maeda S: Genome-wide association studies for life-style related diseases. Clin Calcium 26(3): 419-425, 2016.

11 Yutaka S, Tomoko F and Kohsuke S: DNA polymorphisms. Jpn J Clin Pathol 61(11): 1001-1007, 2013.

12 Park JH, Kim NS, Park JY, Chae YS, Kim JG, Sohn SK, Moon JH, Kang BW, Ryoo HM, Bae SH, Choi GS and Jun SH: MGMT-535G $>$ T polymorphism is associated with prognosis for patients with metastatic colorectal cancer treated with oxaliplatin-based chemotherapy. J Cancer Res Clin Oncol 136(8): 1135-1142, 2010. 
13 Hu Z, Wang H, Shao M, Jin G, Sun W, Wang Y, Liu H, Wang Y, Ma H, Qian J, Jin L, Wei Q, Lu D, Huang W and Shen H: Genetic variants in $M G M T$ and risk of lung cancer in Southeastern Chinese: a haplotype-based analysis. Human mutation 28(5): 431-440, 2007.

14 Garcia-Closas M, Kristensen V, Langerød A, Qi Y, Yeager M, Burdett L, Welch R, Lissowska J, Peplonska B, Brinton L, Gerhard DS, Gram IT, Perou CM, Børresen-Dale AL and Chanock S: Common genetic variation in TP53 and its flanking genes, WDR79 and ATP1B2, and susceptibility to breast cancer. Int J Cancer 121(11): 2532-2538, 2007.

15 Malmer BS, Feychting M, Lönn S, Lindström S, Grönberg H, Ahlbom A, Schwartzbaum J, Auvinen A, Collatz-Christensen H, Johansen C, Kiuru A, Mudie N, Salminen T, Schoemaker MJ,
Swerdlow AJ and Henriksson R: Genetic variation in $p 53$ and ATM haplotypes and risk of glioma and meningioma. $\mathrm{J}$ Neurooncol 82(3): 229-237, 2007.

16 Ogino S, Hazra A, Tranah GJ, Kirkner GJ, Kawasaki T, Nosho K, Ohnishi M, Suemoto Y, Meyerhardt JA, Hunter DJ and Fuchs CS: $M G M T$ germline polymorphism is associated with somatic $M G M T$ promoter methylation and gene silencing in colorectal cancer. Carcinogenesis 28(9): 1985-1990, 2007.

Received April 6, 2017

Revised April 20, 2017

Accepted April 25, 2017 